First publ. in: Physical Review B 64 (2001), Article 104503

\title{
Influence of microscopic defects in type-II superconducting thin films on the magnetic flux penetration
}

\author{
Johannes Eisenmenger* and Paul Leiderer \\ Universität Konstanz, Fachbereich Physik, D-78457 Konstanz, Germany \\ Michael Wallenhorst and Horst Dötsch \\ Universität Osnabrück, Fachbereich Physik, D-49069 Osnabrück, Germany \\ (Received 28 July 2000; revised manuscript received 20 February 2001; published 6 August 2001)
}

\begin{abstract}
The magnetic flux penetration into thin type-II superconducting films with circular defects is investigated. The artificial circular defects (diameter $=40 \mu \mathrm{m}$ ) in an $\mathrm{YBa}_{2} \mathrm{Cu}_{3} \mathrm{O}_{7-\delta}$ thin film (thickness $\approx 300 \mathrm{~nm}$ ) were prepared by pulse-laser irradiation. The flux penetration into the zero-field-cooled superconducting film was visualized by means of the magneto-optic method. A stepwise increase of the external magnetic field allowed a detailed investigation of the influence of local defects on the flux penetration. For a magnetic field parallel to a long sample (longitudinal geometry) with a long cylindrical defect a single parabolic discontinuity line appears. Also in the case of a thin superconducting film exposed to a transverse magnetic field (transverse geometry), a single parabolic discontinuity line has been supposed in the vicinity of a local defect. On the contrary, our investigations show that the flux and current distribution around a single defect in a superconducting thin film can be determined not by a single, but by two discontinuity parabolas. In thin superconducting films in transverse geometry screening currents in the Meissner region $\left(j<j_{c}\right)$ are present in contrast to extended infinitely long samples in the longitudinal geometry. We explain our experimental results by the influence of these Meissner screening currents on the temporal formation of the shape of an approaching flux front.
\end{abstract}

PACS number(s): 74.60.Ec, 74.76.-w, 74.80.-g, 78.20.Ls

\section{INTRODUCTION}

After zero-field cooling (ZFC) a type-II superconductor and the application of an external magnetic field, the penetration of magnetic flux into the sample takes place in several steps. At small magnetic fields Meissner surface currents, flowing within the penetration depth $\lambda$, prevent magnetic flux penetration into the sample. Upon increasing the external magnetic field, the first vortices are formed at the sample edge where the local magnetic field $B_{l o c}$ exceeds the lower critical field $B_{c 1}$ first. The local magnetic field $B_{l o c}$ is the superposition of the external magnetic field and the self-field of the sample produced by the screening current. By increasing the external field the screening current density $j$ increases and locally reaches the critical value $j_{c}$ where the pinning force is balanced by the Lorentz force which drives the vortices towards the center of the sample. Also an enhanced pinning at the sample edge due to barrier effects is possible which plays a dominant role especially in the case of weak volume pinning forces. In the simplest case of an infinitely long hard type-II superconductor in parallel field (longitudinal geometry), without edge barrier effects, and a constant critical current density $j_{c}$ (i.e., independent of the local field) the situation is described by the Bean model ${ }^{1}$ which leads to a constant magnetic-field gradient in the superconductor in the region where the flux has penetrated. In the outer part of the sample, where vortices already penetrated, the critical state (Shubnikov phase) develops. The inner part stays in the Meissner state, where no flux lines are present. The higher the external magnetic field is, the more the Shubnikov region grows at the expense of the Meissner region. In general the flux and current distribution in the superconductor is influenced by the sample geometry, the external magnetic field and the critical current density $\mathbf{j}_{\mathbf{c}}(B, \mathbf{r})$. Penetration of perpendicular flux into thin superconductors has recently been calculated analytically, within the Bean assumption $j \leqslant j_{c}$ and without an influence of edge barrier, for thin circular disks $^{2,3}$ and long stripes ${ }^{4}$ in transverse field, and for strips carrying a transport current, ${ }^{5}$ see also the analytical solution for a strip with bulk pinning and edge barrier. ${ }^{6,7}$ For a negligible edge barrier, a general two-dimensional equation of motion for the sheet current in a thin planar superconductor of arbitrary shape was given by Gurevich and Brandt. ${ }^{8}$ The equation was solved for the cases of quadratic, rectangular samples, ${ }^{8}$ crosslike samples, and quadratic samples with semicircular indents. ${ }^{9}$

A different situation arises if the superconducting thin film contains nonconducting regions which are not connected to the sample edge and which are large enough that they act not solely as a pinning center, but screening currents must flow around them. A model for the current and magnetic flux distribution in the critical state around a circular nonconducting defect in type-II superconductors was given by Campbell and Evetts. ${ }^{10}$ They considered an infinitely long type-II superconductor containing an infinitely long cylindrical defect (Fig. 1). The sample is exposed to a homogeneous external magnetic field parallel to the cylindrical defect and perpendicular to the image plane. In the regions, which are in the critical state already (bright), the assumed Bean model $j_{c}$ $=$ const. and the continuity equation lead to critical current streamlines parallel to the sample edge and the perimeter of the defect. At the dotted parabolic line, the so-called discontinuity line, the critical current density has a very small ra- 


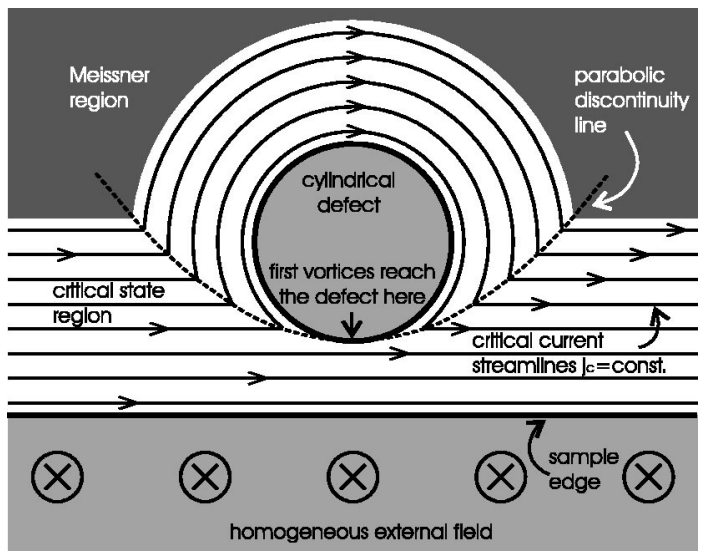

FIG. 1. Current distribution in an infinitely long sample containing a long cylindrical defect. The sample is exposed to a homogeneous external magnetic field parallel to the cylindrical defect and perpendicular to the image plane.

dius of curvature and the external magnetic field is weakened very effectively due to outward bent current paths. The parameter $p$ of the parabolic discontinuity line with the equation $y=x^{2} / 2 p$ corresponds to the defect diameter $2 R$. Since Schuster and co-workers, ${ }^{11,12}$ this model has also been used to describe the influence of circular defects in superconducting thin films. ${ }^{13,14}$ In contrast to the sample geometry considered by Campbell and Evetts, the demagnetizing factor of a thin film cannot be neglected, and screening currents flow not only in the outer parts of the sample which are already in the critical state $\left(j=j_{c}\right)$, but also in the central parts even if they are still in the Meissner state $\left(j<j_{c}\right){ }^{2}$

The question arises how far the Meissner currents influence the final flux distribution near defects in superconducting thin films and whether a modified model has to be used instead of Campbell's and Evetts' model. To answer this question we investigated different states of magnetic flux penetration into type-II superconducting thin films in the presence of well-defined artificial defects.

\section{EXPERIMENTAL TECHNIQUE}

The investigated $\mathrm{YBa}_{2} \mathrm{Cu}_{3} \mathrm{O}_{7-\delta}$ (YBCO) thin film was prepared by pulsed-laser deposition on $r$-plane sapphire (thickness: $1 \mathrm{~mm}$ ) with $\mathrm{CeO}_{2}$ buffer layer. ${ }^{15}$ On the back of the sapphire substrate a second YBCO thin film had been deposited before. Both layers had a thickness of $300 \mathrm{~nm}$ and a critical temperature $T_{c} \approx 89 \mathrm{~K}$. Such samples are very common for the fabrication of coplanar microwave filters where defects with a size of microns can significantly reduce the performance.

The used magneto-optical method is based on the Faraday effect, i.e., the rotation of the polarization plane of linearly polarized light which passes a magneto-optically active layer exposed to the magnetic field of the underlying superconductor. Since the rotation angle depends on the magnetic field one can visualize the flux distribution as optical contrasts in a polarization microscope. For the magneto-optical investigation the sample was zero-field cooled in a continuous flow cryostat which had an optical window. As a

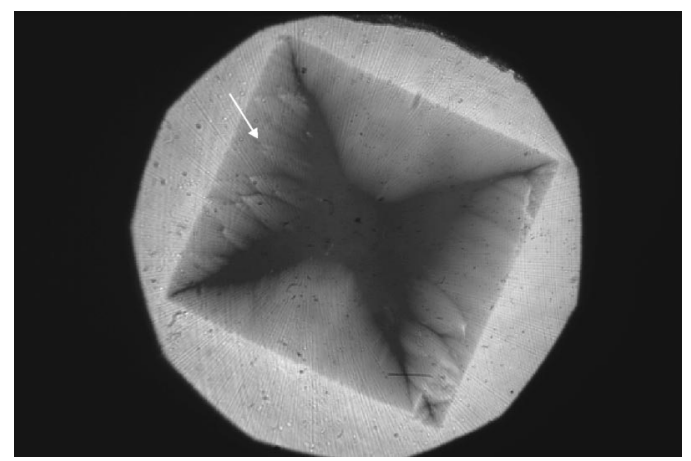

FIG. 2. Magneto-optical image of the magnetic flux distribution of a rectangular YBCO thin film (size $\approx 1 \times 1 \mathrm{~cm}^{2}$ ) at $10 \mathrm{~K}(\mathrm{ZFC})$ and a homogeneous external field of $113 \mathrm{mT}$.

magneto-optical layer placed onto the superconductor we used a doped ferrimagnetic iron-garnet layer grown onto gadolinium-gallium-garnet substrate by liquid phase epitaxy. ${ }^{16}$ The ferrimagnetic domains of the garnet layer with in-plane anisotropy are not visible under the polarization microscope, if illuminated and observed perpendicular to the film surface ${ }^{17}$ To increase the light reflection an aluminum layer is evaporated onto the iron-garnet film that is directed towards the superconductor. The flux distribution was measured with almost crossed polarizer and analyzer, so bright areas indicate a high-flux density component perpendicular to the superconducting layer. For measuring the distribution of the local light intensity we used a 12-bit slow-scan chargecoupled device (CCD) camera.

\section{RESULTS AND DISCUSSION}

Figure 2 shows the magnetic flux distribution of the rectangular YBCO thin film (size $\approx 1 \times 1 \mathrm{~cm}^{2}$ ) at $10 \mathrm{~K}(\mathrm{ZFC})$ and a homogeneous external field of $113 \mathrm{mT}$. The typical magnetic flux distribution of rectangular or quadratic thinfilm samples develop. In a perpendicular external magnetic field flux enters a quadratic or rectangular type-II superconducting thin film preferentially at the edges in the form of a convex flux front, but not from the corners. $8,18,19$

On the right and the left of the sample in Fig. 2 the flux distribution is locally disturbed by natural and artificial defects. Their influence can be better examined at higher magnification. Figure 3 shows the marked region in Fig. 2 at much higher magnification and some lower magnetic field $B_{\text {ext }}=51 \mathrm{mT}$. On the left the flux front is located separating the (overexposed) Shubnikov region, where vortices have penetrated already, from the Meissner region on the right where no flux lines are present. In the Meissner region the influence of four nonconducting circular defects is visible producing at each defect a characteristic black-and-white structure (position 2 and 1). At this stage the flux front has not yet reached the defects, and the regions around the defects are still in the Meissner state. The defects were produced by burning small holes into the YBCO thin film by means of a focused laser beam. The diameter of the holes is only $40 \mu \mathrm{m}$ and the distance between them is about $200 \mu \mathrm{m}$. The origin of the black-and-white structure takes 


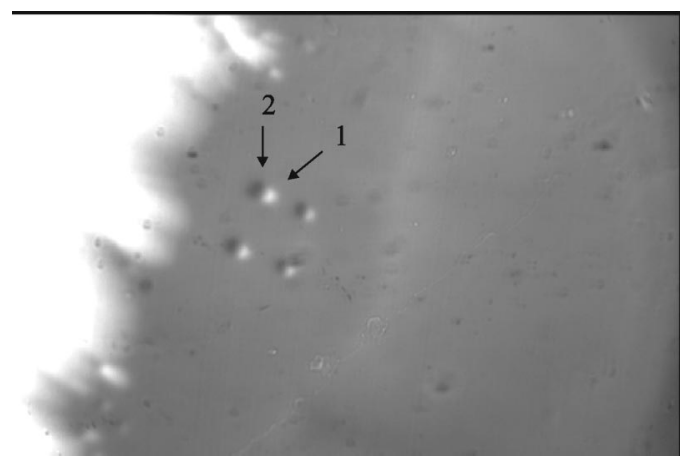

FIG. 3. Flux distribution around four nonconducting round defects (diameter $=40 \mu \mathrm{m})$ at $10 \mathrm{~K}(\mathrm{ZFC})$ and an external magnetic field of $51 \mathrm{mT}$. The distance between the defects is $200 \mu \mathrm{m}$ (after Ref. 20).

place due to the superposition of the external field and of the field produced by Meissner screening currents which are disturbed by the local defects. ${ }^{20,21}$

If a region inside a superconducting thin film is separated from the sample edge by areas, which are still in the Meissner state and having a subcritical current density, the integrated flux through the regarded area has to be zero. Note that flux bundles can cross Meissner regions very suddenly without being permanently pinned if the current density in the Meissner region is overcritical. Schuster et al. ${ }^{22}$ investigated the penetration into $\mathrm{Bi}_{2} \mathrm{Sr}_{2} \mathrm{CaCu}_{2} \mathrm{O}_{8+\delta}$ single-crystal platelet with irradiation-enhanced pinning in the edge zone and could observe this effect on their sample by magnetooptics. This situation is analogous to the penetration of flux bundles over an edge barrier observed in type-I superconductors. ${ }^{23}$ The influence of an edge barrier in hightemperature superconductors (HTSC's) could be observed directly in extremely low pinning $\mathrm{Bi}_{2} \mathrm{Sr}_{2} \mathrm{CaCu}_{2} \mathrm{O}_{x}$ single crystals by magneto-optical observation ${ }^{24}$ and corresponding Hall-probe measurements ${ }^{6}$ of the characteristic free flux penetration to the center of the crystals at $T>35 \mathrm{~K}$. In YBCO there is normally higher pinning which would mask the vortex penetration dynamics associated with the geometrical barrier. Even in very pure detwinned or monotwin domain YBCO single crystals with reduced amount of impurities, resulting in significantly fewer pinning centers, the influence of a geometrical barrier could be observed only for temperatures $T \geqslant 60 \mathrm{~K}^{25,26}$ At lower temperatures $T=40 \mathrm{~K}$ the influence of an edge barrier disappeared. ${ }^{25}$ In our case of a YBCO thin film at $T=10 \mathrm{~K}$ strong pinning is inherent because of interaction of vortices with lattice defects, dislocations, and tensions arising at a film-substrate interface. Due to strong pinning, edge barrier effects can be neglected and current density in the Meissner regions stays always subcritical. Flux penetrates gradually into the thin film from the edge (see Fig. 2) according to the Bean model as is usual in hard type-II superconductors. As flux bundles cannot cross suddenly the Meissner region due to overcritical current densities, the integrated flux through an arbitrary region surrounded by areas in the Meissner state has to be zero if the sample was zero-field cooled before. Such regions are around the defects in Fig. 3, which are characterized by the

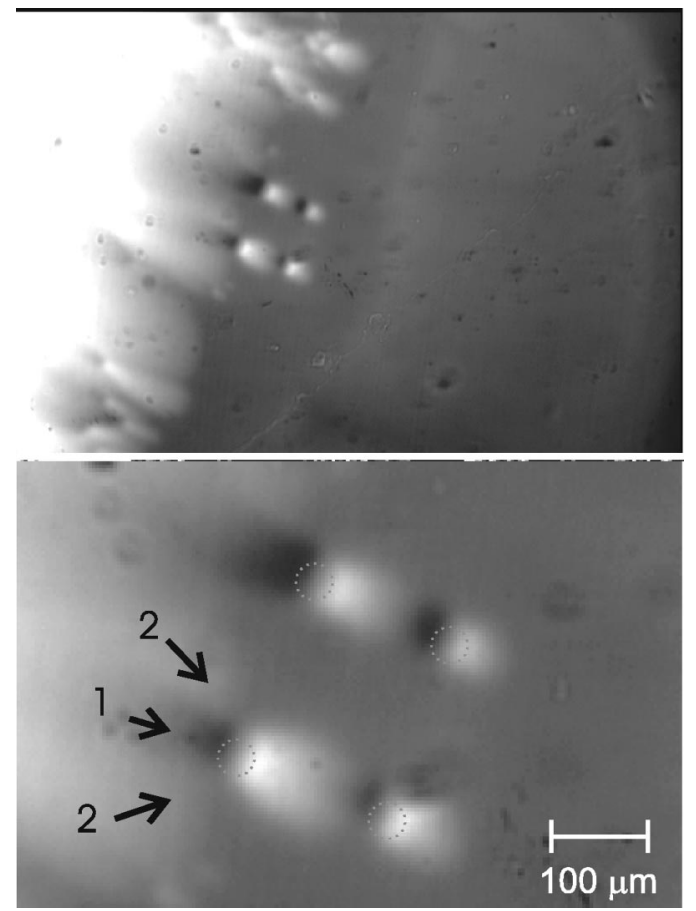

FIG. 4. The defects of Fig. 3 after increasing the external magnetic field to $58 \mathrm{mT}$. The lower image shows details of the image above. Meissner currents, which flow around the defect, cause the flux front to reach the defect at the position 2 earlier than at 1 . The dotted circles indicate the position and diameter of the defects determined with normal optical microscopy.

black-and-white structures, i.e., an area where the normal component of the flux density is negative (position 2) and another one where it is positive (position 1). As the polarizer and analyzer were not totally crossed the sign of the normal flux component can be distinguished.

A slightly different situation occurs at a somewhat higher field $B_{\text {ext }}=58 \mathrm{mT}$ (Fig. 4). The defects to the right are again not reached by the flux front, but on their right-hand side, the side which is oriented towards the sample center, regions with an enhanced normal component of flux density appear. As the extension is significantly larger than the defect, in contrast to the situation in Fig. 3, we assume that these regions are in the critical state. We suggest that at the righthand side of the defects the screening current density reached the critical value $j_{c}$ and vortices partly penetrate through the adjacent thin film as sketched in Fig. 5. The integrated normal component of the flux through the area of the defect and the region nearby the defect, where flux partly penetrates through the film, still remains zero. Apart from flux lines which penetrate through the film on the right-hand side of the defect, the situation sketched in Fig. 5 agrees with the current and field distribution around local defects as in Fig. 3 where the flux front is still far apart. In the later case the black-and-white structure is very symmetric and more restricted to the region of the defect, because flux lines do not penetrate through film area adjacent to the defect. ${ }^{20,21}$

Much closer to the flux front are the two defects on the left in Fig. 4. Applying Campbell's and Evetts' model to a defect in a thin film, as done in Refs. 11-14, we would 


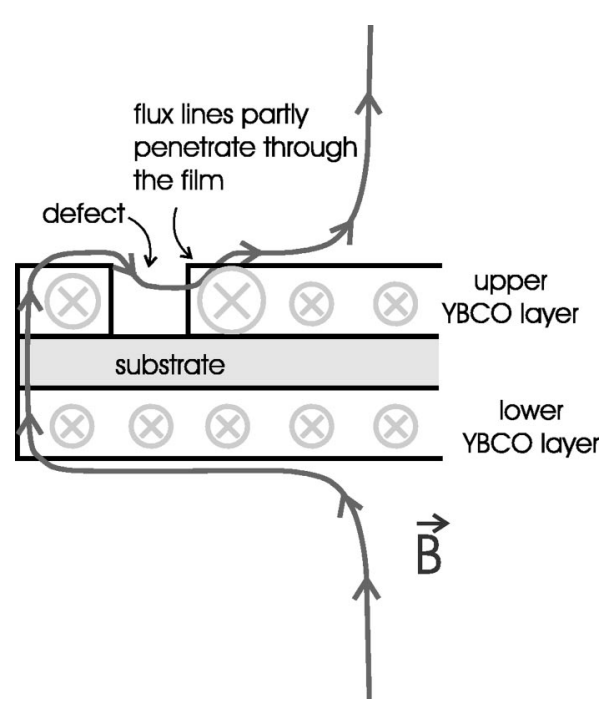

FIG. 5. Sketch of a defect which is separated from the sample edge by Meissner regions, similar to the situation of the defects on the right in Fig. 4(b). In contrast to the flux distribution around the defects in Fig. 3, the screening currents reached their critical value on the defect side which is oriented towards the center of the sample (to the right), so flux penetrates partly through the adjacent thin film. The sketch is not to scale (thickness of YBCO layer $\approx 10^{-3} \times$ thickness of the substrate).

expect that the flux front reaches a defect at the vertex of the parabolic discontinuity line (see Fig. 1). Schuster et al. call it the intersection point of the $d^{+}$and $d^{-}$line. ${ }^{11,12}$ There the first flux lines should penetrate through the defect and the integral flux through the defect area is no longer zero. In contrast Campbell's and Evetts' model seems not suitable to describe our observation of flux penetration into defects of thin films. At the lower defect on the left in Fig. 4 we observe clearly that the flux front does not reach the defect at the assumed position 1 first, but on the side of it at position 2 where vortices will reach the defect first. Moreover the flux front is hindered to penetrate the defect at position 1 .

One might suppose that this discrepancy to Campbell's and Evetts' model is due an influence of the second YBCO layer on the back of the sample which is not faced to the magneto-optical layer, but exposed to the external magnetic field as well. This interpretation is at variance with the fact that this form of flux front, and the following at higher external fields, has been observed not only for double-sided YBCO thin films, but also for isolated defects on YBCO thin films where only one side of the substrate was coated. ${ }^{27}$ In the case of a double-sided YBCO thin film the imaged flux distribution at the upper YBCO layer, which is faced to the magneto-optical garnet layer, is influenced by the current density in the lower YBCO layer only by an averaged current distribution. The area, over which is averaged, is determined by the $1 \mathrm{~mm}$ thick sapphire substrate. A homogeneous second YBCO thin film on the back causes an enhanced screening of the sample in general, but does not influence local variation of the flux front form on the scale of a few $10 \mu \mathrm{m}$ as relevant for the above discussion of Fig. 4.

In the case of an infinitely long sample in parallel magnetic field the screening currents exist only between the flux

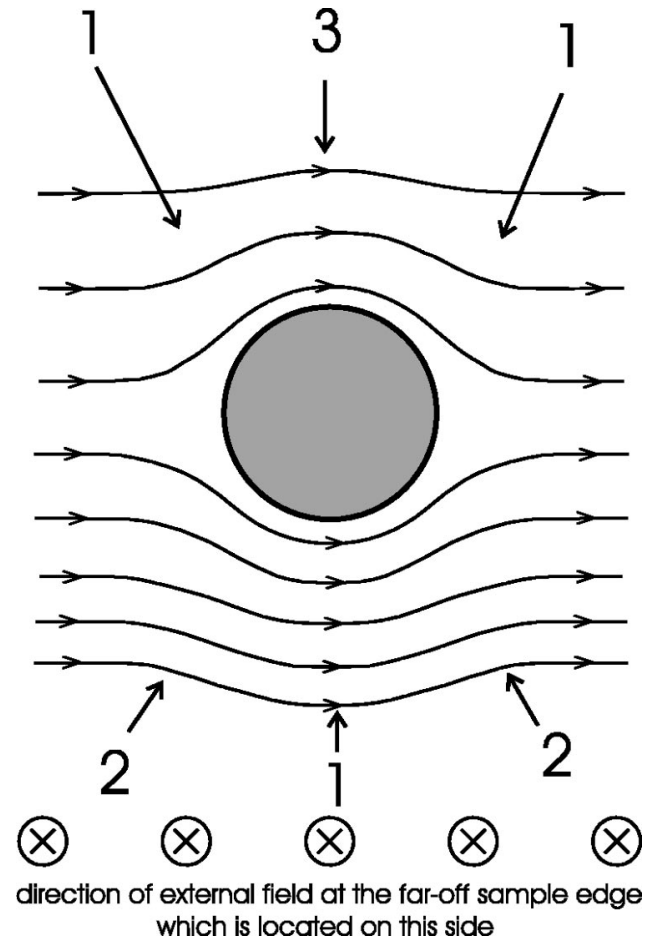

FIG. 6. Current distribution of the Meissner currents in a thin film with a circular defect, if the flux front, which comes from the bottom, is relatively far away. The sample is exposed to a homogeneous external magnetic field perpendicular to the image plane.

front and the sample edge, and the current streamlines are exactly parallel to the sample edge till the flux front reaches the defect at the vertex of the parabolic discontinuity line in Fig. 1. In contrast screening currents in thin films flow also in regions which are still in the Meissner state. ${ }^{2}$ With a film thickness on the order of the penetration depth the screening currents flow not only on the surface, but within the entire film thickness. Figure 6 clarifies the current streamlines of Meissner currents $\left(j<j_{c}\right)$ around a defect. For this sketch it was assumed that the flux front is still relatively far away and its form is more or less straight. When the external field is further increased and the flux front comes closer to the defect, more and more flux components oriented perpendicular to the film must be screened. In the regions 2 and 3 these perpendicular field components can be screened less effectively because the current streamlines are bent inwards (with respect to the center of the sample), in contrast to the regions 1 where the external magnetic field can be screened more effectively due to current streamlines bent outward. Therefore with increasing external field, the initial current distribution of Fig. 6 changes to a situation where the local screening current density has to adjust in the regions 2 and 3 to higher values than in the regions 1 . Since the critical current density is reached at the regions 2 earlier than at the regions 1, the flux front moves from the regions 2 faster towards the defect. In the regions 1 the critical current density is reached much later, because there even a relatively low current density can screen the perpendicular field components very effectively. Due to this bending of Meissner currents the final current streamlines in the critical state are 


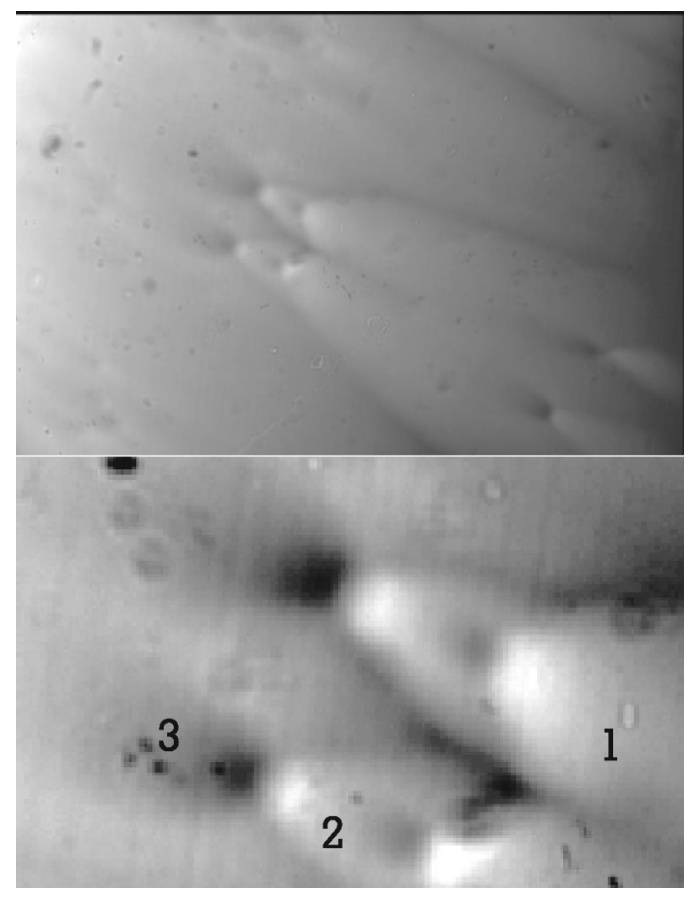

FIG. 7. The defects of Fig. 3 after increasing the external magnetic field to $82 \mathrm{mT}$. The lower image shows details of the image above. Besides the parabolic discontinuity lines 1 and 2 oriented towards the center of the sample, the left defects show an additional discontinuity line 3 oriented towards the edge of the sample. The contrast of the lower image was enhanced in comparison to the upper image.

also bent and the flux front loses its originally straight form which it had far away from the defect.

In Fig. 7 the external field was increased further to $82 \mathrm{mT}$. The entire imaged area is now in the Shubnikov state. Similar to the model of Campbell and Evetts, areas with slightly increased flux density develop on the side of the four defects which is oriented towards the center of the sample. These areas are bordered by dark parabolic discontinuity lines. In contrast to Campbell's and Evetts' model, the two defects on the left show a second parabolic discontinuity line which is oriented towards the edge of the sample. The area bordered by this discontinuity line shows a slightly decreased instead of increased flux density. In contrast, at the right defects a second discontinuity line does not develop. The flux distribution around the right defects, which is discussed below, is strongly influenced by the left defects which were reached by the flux front before.

Let us consider the left defects first. Since the above discussed influence of a defect on the direction of the Meissner currents and the influence of the Meissner currents on the advancing flux front (Fig. 6), we expect that a current distribution like in Fig. 6 develops to a current distribution in the critical state very similar to the one in Fig. 8. As in Fig. 6 this model requires that the flux front had a straight form, when it was far away from the defect. Comparable with the model of Campbell and Evetts a constant critical current density, i.e., equidistant current streamlines, is supposed. In contrast to their model (Fig. 1), the critical current streamlines are parallel to the perimeter of the defect even on the side which is

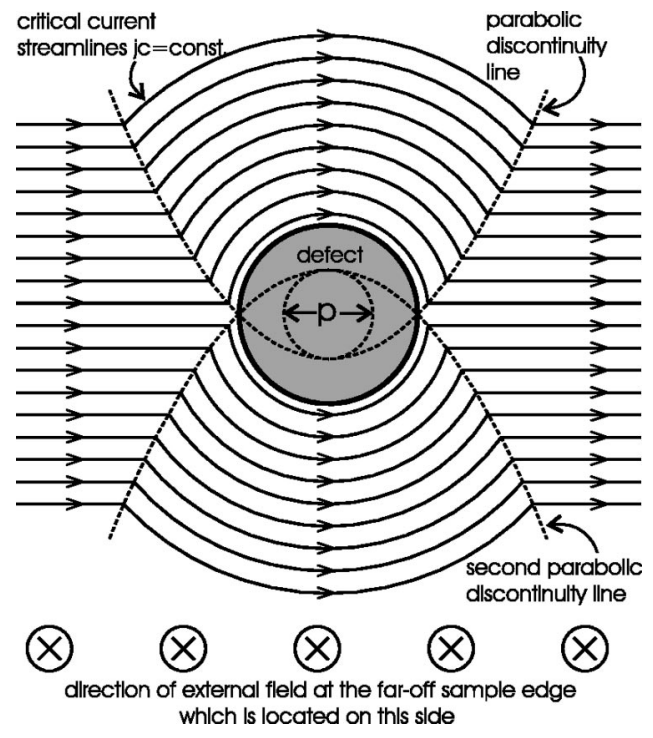

FIG. 8. Critical current distribution around a circular defect (dark) in a superconducting thin film, which develops if the flux front had a straight form, when it was far away from the defect. In contrast to the longitudinal geometry, a second discontinuity parabola develops. The parameters $p$ of the parabolas correspond to the defect radius.

oriented to the edge of the sample. This is not in contradiction to a parallel current flow along a straight edge of a sample, as long as the edge is far enough away from the defect. For large distances from the defect, a straight flux front was assumed which is consistent with the diminishing curvature of the concentric critical current streamlines. The very symmetric current distribution in Fig. 8 explains the field enhancement towards the center of the sample as well as the field decrease towards the edge. The symmetry is reflected in the two discontinuity lines. According to our model the discontinuity lines are described by the equation

$$
y= \pm\left[\frac{x^{2}}{2 p}-\frac{p}{2}\right],
$$

at which the origin of the cartesian coordinate system is the center of the defect and the parabolic parameter $p$ corresponds to the radius $R$ of the defect (dark area). In Fig. 9 the squares correspond to the measured form of the discontinuity lines of the lower defect on the left in Fig. 7. Fitting Eq. (1) to these points leads to two parabolas with identical parameter $p=20.8 \pm 0.2 \mu \mathrm{m}$. These values correspond very well to the actual radius $R=20 \mu \mathrm{m}$ of the defect determined with normal light microscopy. The slightly different radius could be due to reduced superconducting properties at the edge of the defect which cannot be distinguished from undisturbed superconducting regions with normal light microscopy. The model of Campbell and Evetts, which was also used to estimate the radius of defects in thin films, ${ }^{14}$ would lead to a radius $\widetilde{R}=p / 2$ which is half as wide (see small dashed circle in Fig. 8). ${ }^{29}$

The excellent agreement between the measured discontinuity lines and the fitted parabolas a posteriori justifies a symmetric current distribution and our assumption $j$ 


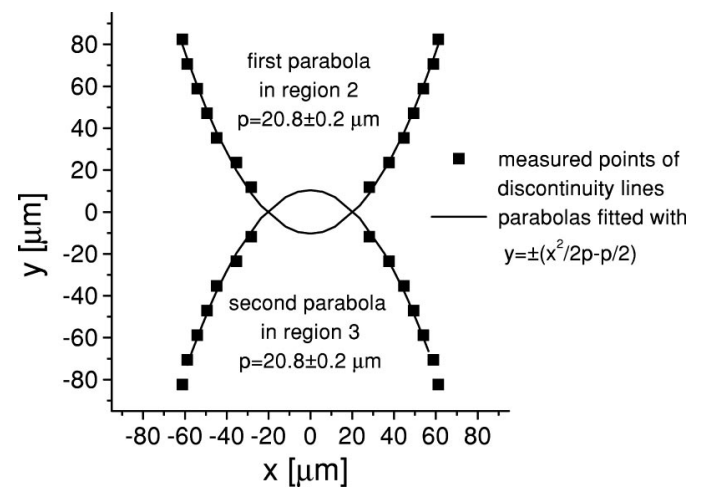

FIG. 9. Squares: measured form of the discontinuity lines of the lower defect on the left in Fig. 7 (areas 2 and 3 in the detailed figure). Solid lines: measured discontinuity lines fitted with Eq. (1) lead to parabolas with identical parameter $p=20.8 \pm 0.2 \mu \mathrm{m}$.

$=j_{c}(B)=$ const in the vicinity of the defect. In the case of a significant influence of the local magnetic inductance $B$ on the critical current density $j_{c}$ we would expect a deviation from the pure parabolic form and a difference between the two discontinuity lines oriented towards the center and the edge of the sample. Since such deviations are not observed we assume that a strong local-field dependence of $j_{c}$, from which Jooss et al. ${ }^{14}$ started out to interpret the flux distribution near a defect in a superconducting disk, is not required in our case.

Let us now consider the defects to the right in Fig. 7. These defects are not accompanied by two discontinuity parabolas, but only by the one oriented towards the center of the sample. As the flux front, which approaches the right defects, is strongly influenced by the left defects which are very close and were reached before, the flux front cannot be assumed straight for large distances. In particular, this can be observed in Fig. 4. The enhanced flux density on the righthand side of the left defects is accompanied by current streamlines which are strongly bent towards the center of the sample. On the other hand, the Meissner screening currents on the left-hand side of the right defects have the tendency to bend towards the edge of the sample. During further penetration of the flux front these two opposite bends could cancel each other resulting in a current distribution which cannot be described by Fig. 8, but is better approximated by the model of Campbell and Evetts. The wider discontinuity parabola with identical defect radius $(R=20 \mu \mathrm{m})$ and the missing second discontinuity parabola are consistent with this interpretation. As mentioned above, for a current distribution identical to the one in the model of Campbell and Evetts the parameter $p$ of the parabola would be equal to the defect diameter, i.e., twice as big as for the current distribution in Fig. 8. Since the measured $p$ of the discontinuity parabolas of the right defects are somewhat smaller than the defect diameter, and since there is a slight reduction in the flux density on the left-hand side of the right defects in Fig. 7, the model of Campbell and Evetts is only a good approximation. The true current distribution around these defects lies between the distribution given by Campbell and Evetts and the one in Fig. 8. Similar effects are expected if the considered defect is located very close to the sample edge. Current streamlines parallel to a sample edge, which is very close to the defect, prevent the undisturbed development of the flux distribution in Figs. 6 and 8. This could be a reason for observing only a single discontinuity parabola in thin films in the past. ${ }^{11-14}$

Apart from the discussed four defects in the center of Fig. 7 , there are two additional ones located on the far lower right-hand side of the upper figure (prepared in the same manner). Again two discontinuity parabolas are observed at each defect, because the four central defects are so far away that the approximation of a straight flux front for large distances is valid again. There the parameter $p$ of the parabolas again corresponds to a defect radius of $20 \mu \mathrm{m}$.

It would be interesting to confirm our qualitative model by more exact calculations. Especially if they are able taking into account the influence of possible defects nearby or smaller distances between defect and edge of the sample. Recently Gurevich et al. calculated the steady-state current flow perpendicular to a thin nonconducting strip in an infinite media by hodograph transformation of the equations $\nabla \times \mathbf{E}$ $=0, \nabla \times \mathbf{H}=\mathbf{j} .{ }^{28}$ For $j<j_{c}$, the $E-j$ curve of the superconducting media is approximated by the power-law dependence $E=E_{c}\left(j / j_{c}\right)^{n}$ with $n \gg 1$ for magnetic field $\mathbf{H}$ below the irreversibility field. Because the boundary conditions of curved boundaries become nonlinear in the hodograph space, a geometry of a cylindrical or circular defect, as in our case, was not solved. Nevertheless, due to the chosen symmetrical boundary conditions a symmetrical current distribution with discontinuity lines on both sides of the nonconducting strip was calculated. In contrast to Fig. 8, the geometry of the nonconducting strip leads to discontinuity lines which are straight near the defect and become parabolic only far away from the defect. The approximation of the $E-j$ curve by the power-law dependence $E=E_{c}\left(j / j_{c}\right)^{n}$ allows to calculate the current distribution not only for the critical state model which is approximated in the limit $n \rightarrow \infty$, but also for the more realistic case of finite $n$. For the later case discontinuity lines with a finite width are found similarly to former calculations of the current distribution in quadratic and rectangular samples. ${ }^{8}$ The width is determined by the curvature of the current-voltage law $E(J)$ near $j=j_{c}$, i.e., the exponent $n$ in the model $E=E_{c}\left(j / j_{c}\right)^{n}$, and decreases with increasing $n$ and decreasing distance from the defect. In the context of these calculations Fig. 8 is only an approximation for $n \rightarrow \infty$. Unfortunately, up to now the geometry of a circular defect was not solved for the more realistic approximation $E$ $=E_{c}\left(j / j_{c}\right)^{n}$ with finite $n$. Moreover, the solution in Ref. 28 is a steady-state solution where geometrical boundary conditions have to be given a priori. To prove the temporary formation of the current distribution with the final distribution in Fig. 8 a solution of the time-dependent Maxwell's equations would be necessary. Time-dependent solutions were given for superconducting thin films with different shapes of boundaries. ${ }^{8,9}$ Unfortunately, only the sample boundaries have been varied and geometries of nonconducting areas within a superconducting media have not been considered.

\section{CONCLUSION}

By means of the magneto-optic technique we have visualized the flux distribution around different defects in a 
YBCO thin film. The stepwise increase of the external magnetic field allows us to investigate in detail how a magnetic flux front advances on circular defects and what current distribution in the final critical state develops.

It could be experimentally observed that the magnetic flux distribution around a circular defect in a superconducting thin film cannot be described by a model which was often used in the case of thin films, ${ }^{11-14}$ though it was originally reduced by Campbell and Evetts for a cylindrical defect in an infinitely long sample. ${ }^{10}$ We explain our observation by screening currents in the Meissner region $\left(j<j_{c}\right)$ which are present in thin superconducting films exposed to a perpendicular magnetic field in contrast to infinitely long samples exposed to a parallel field. Screening currents in the Meissner region have to flow around local, nonconducting defects. They lead to a field distortion which locally enhances or weakens the flux penetration and influences the final current distribution in the critical state. The suggested model explains how the flux front advances on a defect and under what circumstances not a single but two discontinuity parabolas appear. Further it becomes evident that in general the flux and current distribution around a defect is not only determined by its own geometry, but can also be influenced by the current distribution around other defects nearby which have been reached by the flux front earlier.

\section{ACKNOWLEDGMENTS}

The authors would like to thank M. Lorenz for providing the YBCO film, Ch. Neumann for sample preparation and U. Bolz, B.-U. Runge, M. Kuhn, and Ch. Jooss for helpful discussions.
*Corresponding author. Permanent address: Universität Ulm, Abteilung Festkörperphysik, D-89069 Ulm, Germany. Email address: johannes.eisenmenger@physik.uni-ulm.de

${ }^{1}$ C.P. Bean, Phys. Rev. Lett. 8, 250 (1962); Rev. Mod. Phys. 36, 31 (1964).

${ }^{2}$ P.N. Mikheenko and Y.E. Kuzovlev, Physica C 204, 229 (1993).

${ }^{3}$ J. Zhu, J. Mester, J. Lockhart, and J. Turneaure, Physica C 212, 216 (1993); J.R. Clem and A. Sanchez, Phys. Rev. B 50, 9774 (1994).

${ }^{4}$ E.H. Brandt, M. Indenbom, and A. Forkl, Europhys. Lett. 22, 735 (1993).

${ }^{5}$ W.T. Norris, J. Phys. D 3, 489 (1970); G.W. Swan, J. Math. Phys. 9, 1308 (1968); E.H. Brandt and M. Indenbom, Phys. Rev. B 48, 12893 (1993); E. Zeldov, J.R. Clem, M. McElfresh, and M. Darwin, ibid. 49, 9802 (1994).

${ }^{6}$ E. Zeldov, A.I. Larkin, V.B. Geshkenbein, M. Konczykowski, D. Majer, B. Khykovich, V.M. Vinokur, and H. Strikman, Phys. Rev. Lett. 73, 1428 (1994).

${ }^{7}$ E. Zeldov, A.L. Larkin, M. Konczykowski, B. Khaykovich, D. Majer, V.B. Geshkenbein, and V.M. Vinokur, Physica C 235240, 2761 (1994); M. Benkraouda and J.R. Clem, Phys. Rev. B 53, 5716 (1996); 58, 15103 (1998).

${ }^{8}$ A. Gurevich and E. H. Brandt, Phys. Rev. Lett. 73, 178 (1994); E. Brand, ibid. 74, 3025 (1995); E.H. Brandt, Phys. Rev. B 52, 15442 (1995).

${ }^{9}$ T. Schuster, H. Kuhn, E. H. Brandt, M. V. Indenbom, M. Kläser, G. Müller-Vogt, H.-U. Habermeier, H. Kronmüller, and A. Forkl, Phys. Rev. B 52, 10375 (1995); T. Schuster, H. Kuhn, and E. H. Brandt, ibid. 54, 3514 (1996).

${ }^{10}$ A.M. Campbell and J.E. Evetts, Critical Currents in Superconductors (Taylor \& Francis, London, 1972); Adv. Phys. 21, 199 (1972).

${ }^{11}$ T. Schuster, Ph.D. thesis, Universität Stuttgart, 1993.

${ }^{12}$ T. Schuster, M.V. Indenbom, M. R. Koblischka, H. Kuhn, and H. Kronmüller, Phys. Rev. B 49, 3443 (1994).

${ }^{13}$ M.R. Koblischka and R.J. Wijngaarden, Supercond. Sci. Technol. 8, 199 (1995); M.R. Koblischka, ibid. 9, 271 (1996).

${ }^{14}$ C. Jooss, R. Warthmann, A. Forkl, and H. Kronmüller, Physica C 299, 215 (1998).

${ }^{15}$ M. Lorenz, H. Hochmuth, D. Natusch, H. Börner, G. Lippold, K.
Kreher, and W. Schmitz, Appl. Phys. Lett. 68, 3332 (1996).

${ }^{16}$ M. Wallenhorst, Ph.D. thesis, Universität Osnabrück, 1998; J. Eisenmenger, S. Kambach, S. Saleh, A. Tihi, P. Leiderer, M. Wallenhorst, and H. Dötsch, J. Low Temp. Phys. 105, 1123 (1996).

${ }^{17}$ L.A. Dorosinskii, M.V. Indenbom, V.I. Nikitenko, Yu. A. Ossip'yan, A.A. Polyanskii, and V.K. Vlasko-Vlasov, Physica C 203, 149 (1992).

${ }^{18}$ R.P. Huebener, V.A. Rowe, and R.T. Kampwirth, J. Appl. Phys. 41, 2963 (1970); V.A. Rowe, R.P. Huebener, and R.T. Kampwirth, Phys. Status Solidi A 4, 513 (1971).

${ }^{19}$ P. Brüll, D. Kirchgässner, and P. Leiderer, Physica C 182, 339 (1991); T. Schuster, M. Leghissa, M.R. Koblischka, H. Kuhn, H. Kronmüller, and G. Saemann-Ischenko, Physica C 203, 203 (1992).

${ }^{20}$ J. Eisenmenger, J. Schiessling, U. Bolz, B.-U. Runge, P. Leiderer, M. Lorenz, H. Hochmuth, M. Wallenhorst, and H. Dötsch, IEEE Trans. Appl. Supercond. 9, 1840 (1999).

${ }^{21}$ M. Baziljevich, T.H. Johansen, H. Bratsberg, Y. Shen, and P. Vase, Appl. Phys. Lett. 69, 3590 (1996).

${ }^{22}$ T. Schuster, M.V. Indenbom, H. Kuhn, E.H. Brandt, and M. Konczykowski, Phys. Rev. Lett. 73, 1424 (1994); T. Schuster, H. Kuhn, E.H. Brandt, M. Indenbom, M.R. Koiblischka, and M. Konczykowski, Phys. Rev. B 50, 16684 (1994).

${ }^{23}$ R.P. Huebener, R.T. Kampwirth, and J.R. Clem, J. Low Temp. Phys. 6, 275 (1972); H. Castro, B. Dutoit, A. Jacquier, M. Baharami, and L. Rinderer, Phys. Rev. B 59, 596 (1999).

${ }^{24}$ M.V. Indenbom, H. Kronmüller, T.W. Li, P.H. Kes, and A.A. Menovsky, Physica C 222, 203 (1994); M.V. Indenbom, G. D'Anna, M.O. Andre, W. Benoit, H. Kronmüller, T.W. Li, and P.H. Kes, in Proceedings of the 7th International Workshop on Critical Currents in Superconductors, 1994, edited by H. W. Weber (World Scientific, Singapore, 1994), p. 327.

${ }^{25}$ M.V. Indenbom, C.J. Van der Beeck, V. Berseth, M. Konczykowski, F. Holtzberg, and W. Benoit, Czech. J. Phys. 46, 1541 (1996).

${ }^{26}$ M.V. Gardner, S. A. Govorkov, R. Liang, D.A. Bonn, J.F. Carolan, and W.N. Hardy, J. Appl. Phys. 83, 3714 (1998).

${ }^{27}$ J. Eisenmenger, Ph.D. thesis, Universität Konstanz, 1999.

${ }^{28}$ A. Gurevich and J. McDonald, Phys. Rev. Lett. 81, 2546 (1998); 
A. Gurevich and M. Friesen, Phys. Rev. B 62, 4004 (2000).

${ }^{29}$ The two parabola equations given on page 227 in Ref. 14 are not consistent with their sketched parabola in Fig. 10. The missing factor 2 compared to the consistent equation in the cited Ref. 12, $r=\left(2 R_{d e f}\right) /[1-\sin (\theta)]$ or $y=x^{2} /\left(4 R_{d e f}\right)-R_{\text {def }}$, respectively, is due to an erratum. The given defect radii were determined with this consistent equation from Ref. 12 [Ch. Jooss (private communication)]. 